\title{
Bisphosphonates Induced Atypical Femoral Fracture in Metastatic Breast Cancer: Misleading Benign Problem in a Malignant Condition
}

\author{
Islam Mubark, Mosab Elgalli, Keith Hayward \\ Department of Orthopaedics Surgery, Burton Queen's Hospital, United Kingdom.
}

\begin{abstract}
Corresponding Author:
Dr. Islam Mubark

Email: islam.mokhtar81@gmail.com

This is an Open Access article distributed under the terms of the Creative Commons Attribution License (creativecommons.org/ licenses/by/3.0).
\end{abstract}

Received Accepted

Published

May 14, 2017

September 5, 2017

September 30, 2017

\begin{abstract}
Background: Diagnosing bisphosphonates induced atypical femoral fracture (AFF) in patient with bony metastasis can be challenging and overlooked. Case Report: We present a case of atypical sub-trochanteric femoral fracture in a patient treated successively with Zoledronic acid and Denosumab with known skeletal metastasis from breast carcinoma. The fracture was fixed using cephalo-medullary nail. Conclusion: Clinical oncologists should consider the possibility of AFFs in patients on long-term bisphosphonates treatment for bone metastases. Inability to identify those lesions as different from original malignant condition can lead to delayed diagnosis, sub-optimal treatment and missing opportunity to prevent progression of incomplete to complete fractures.
\end{abstract}

Keywords: Bone Neoplasms, Breast Neoplasms, Diphosphonates, Denosumab, Femoral Fractures.

\section{Introduction}

We present a case of atypical sub-trochanteric femoral fracture in a patient treated successively with Zoledronic acid and Denosumab with known skeletal metastases from breast carcinoma.

\section{Case Report}

A 70 years old woman was referred to our orthopaedic service from oncology department for atypical fracture of right proximal femur. She was diagnosed with breast carcinoma 10 years back in January 2006 and was treated initially with modified radical mastectomy, post-operative radiotherapy and estrogen receptor antagonist. Her follow up was disease free until four years later in June 2010 when she had multiple pains in lower back and left shoulder. Her bone scan at that time showed multiple lumbar, ribs and shoulder metastases [Fig.1]. She was started initially on Zoledronic acid $4 \mathrm{mg}$ monthly infusions and Herceptin for five years. Her symptoms have improved much and her follow up bone scan in August 2015 showed stable skeletal disease. Shortly after, she started complaining of pain in right upper thigh and new bone scan in October 2015 showed new hot lesion in right proximal femur [Fig.2]. Initially X-rays were not done as this new lesion was presumed a new metastatic focus and patient was continued on Zoledronic acid treatment. X-rays done later on November 2015 as routine follow up showed thickened lateral femoral cortex and was reported as possible osteoplastic metastatic lesion [Fig.3]. Because of worsening thigh pain, patient was started on fractionated doses of radiotherapy to proximal femur in March 2016. Because of its side effects, Zoledronic acid was switched to Denosumab in June 2016 (120 mg subcutaneously every 4 weeks). Her follow up scan six months later in December 2016 showed all skeletal metastasis to be still quiescent apart from the upper femur lesion which became hotter and with transverse like band configuration. At that point a repeat X-rays showed insufficiency fracture along the lateral cortex of the proximal femur [Fig.4]. At that stage patient could only mobilize with two crutches. 


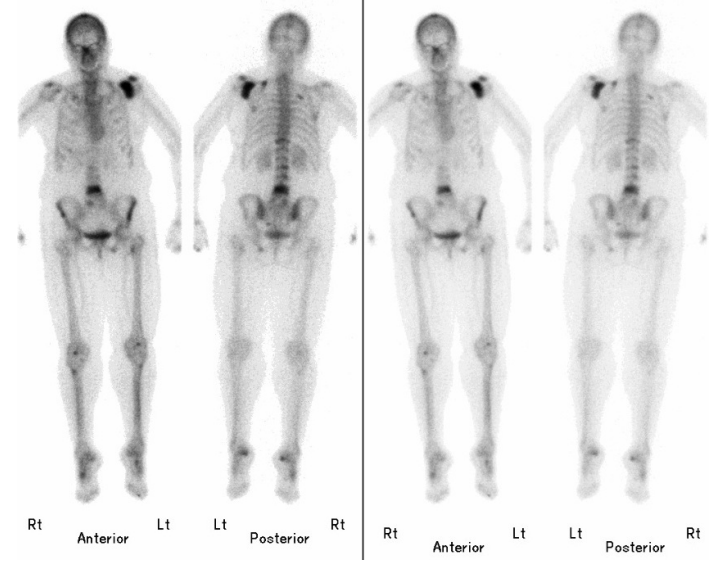

Fig.1: Bone scan in June 2010 showing skeletal metastases involving the lumbar spine, ribs and left shoulder.

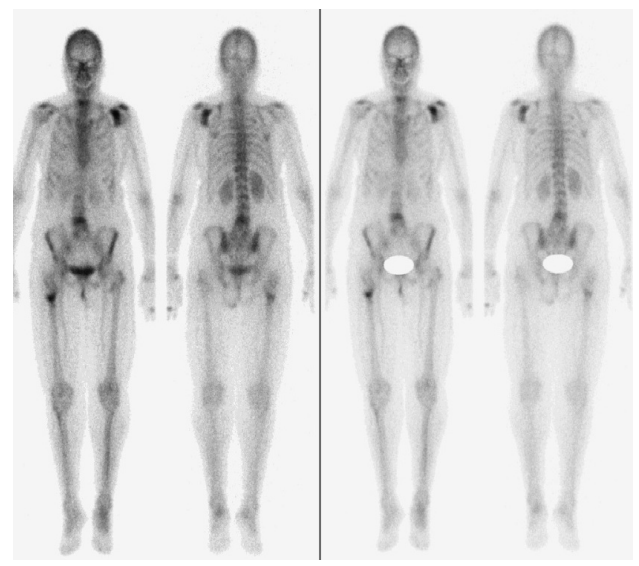

Fig.2: Bone scan in October 2015 showing new hot lesion in right proximal femur.

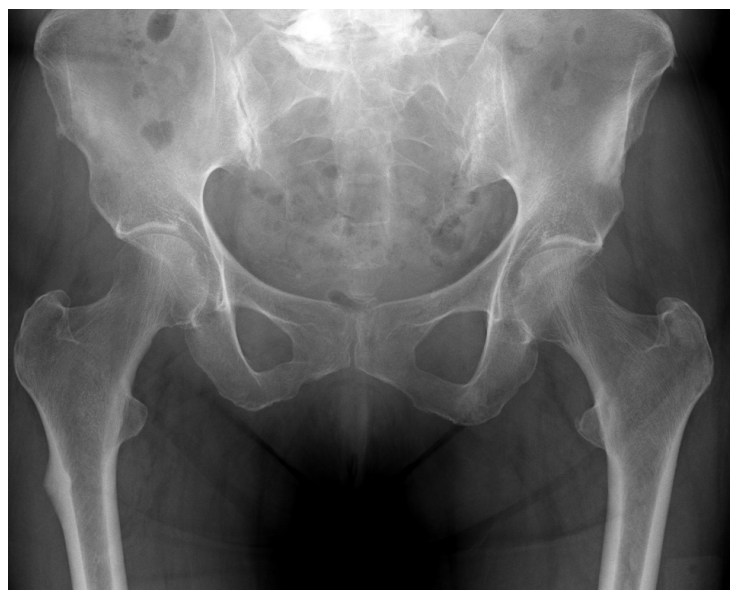

Fig.3: X-ray showing lateral cortex thickening in right femur that was reported as possible osteoplastic bone metastasis.

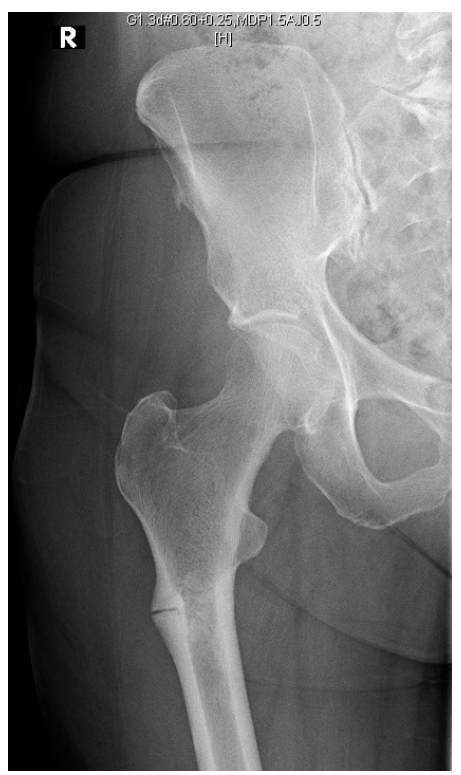

Fig.4: X-ray right femur showing atypical femoral fracture in sub-trochanteric region with characteristics transverse orientation and cortical thickening.

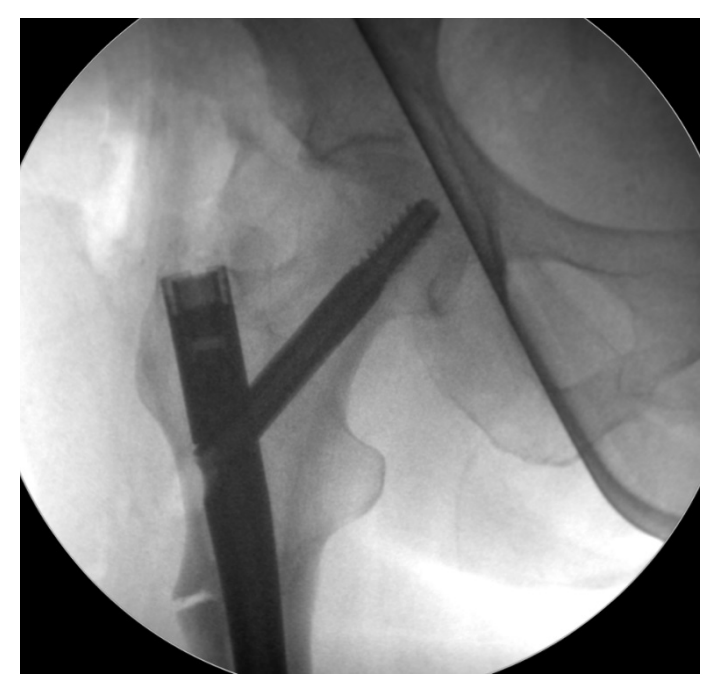

Fig.5: Intra-operative fluroscan image of cephalo-medullary nail fixation of fracture.

The patient was referred to orthopaedic clinic for consultation and initial diagnosis of Bisphosphonate induced fracture was made based on the X-rays criteria of the fracture, the clinical presentation of prodromal symptoms and long history of Bisphosphonate treatment. The decision was to fix it using cephalo-medullary nail [Fig.5] with biopsies taking form fracture site via 
long spoon curette prior to reaming and send for pathology. The pathology came as lamellar bone and marrow with no inflammation, granulomas or evidence of metastatic carcinoma. To assist with fracture healing the Denosumab was stopped preoperatively and patient was started on supplemental calcium and vitamin D. The patient was allowed full weight bearing immediately post-operatively. At 16 weeks follow up, the patient reported no pain at fracture site, she was able to walk without aids and her X-rays showed bridging callus across fracture site [Fig.6a,b].

\section{Discussion}

Bisphosphonates are becoming the first line treatment in many disorders involving increased bone resorption [1]. Zoledronic acid is a potent Bisphosphonate that has been used for the treatment of patients with osteolytic lesions secondary to metastatic bone disease. The American Society of Clinical Oncology recommends the intravenous administration of Zoledronic acid (4 mg) every 3 to 4 weeks for patients with radiologic evidence of bone destruction [2]. Denosumab has a similarly high anti-resorptive capacity as Zoledronic acid and in patient not tolerating Bisphosphonates, it may be a suitable alternative therapy. Atypical femur fractures have been reported after use of Bisphosphonate such as Alendronic acid, but on rare occasion, there have been reports of similar fractures after administration of Zoledronic acid and less common after Denosumab [3]. Zoledronic acid belongs to the nitrogen containing Bisphosphonates and it carries its action via inhibiting osteoclast Farnesyl Pyrophosphate synthase enzyme, required in Mevalonate pathway with net result of inhibiting GTPase formation in osteoclastic cells [4]. Whereas Denosumab is a human monoclonal antibody to the Receptor Activator of Nuclear factor- $\kappa$ B Ligand (RANKL) that prevents the interaction of RANKL with RANK on surface of osteoclasts thereby inhibiting the function of osteoclasts [5].
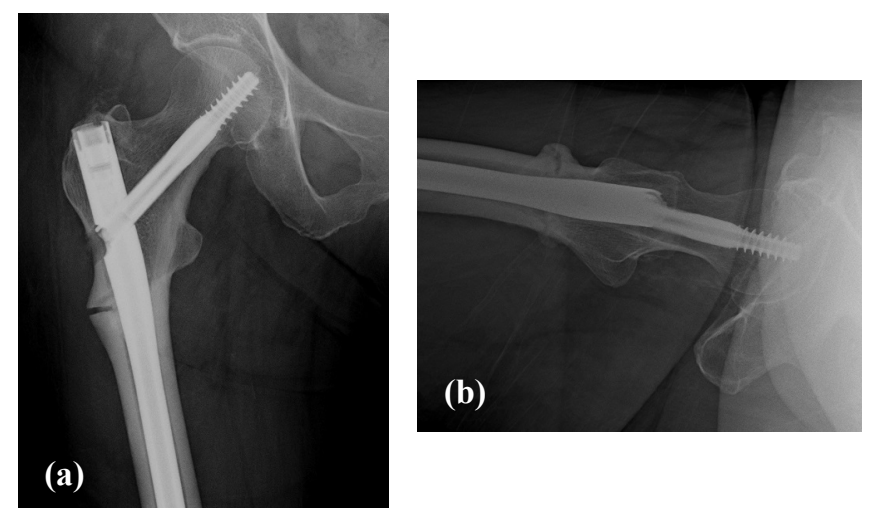

Fig.6(a): Post-operative follow up X-ray at 4 months showing callus formation across fracture site more appreciated on lateral views. (b): Post-operative follow up X-ray at 4 months showing callus formation across fracture site more appreciated on lateral views.

Stress fractures observed with antiresorptive drug treatment are thought to start by accumulation of microscopic fractures. Such micro-fractures formation is a part of bone remodeling. These areas with micro-fractures are normally resorbed by osteoclasts and replaced with new bone by osteoblasts. If this remodeling process is interrupted by anti-resorptive drugs, microfractures will grow, fuse, and cause stress fractures [6]. The risk of AFFs is reported to increase with the increased duration of Bisphosphonate treatment, from 1.8 cases per $10^{5}$ users per year of exposure in the first 2 years, to 113 cases per $10^{5}$ users per year at 8 years of Bisphosphonate use [7]. Our patient developed her fracture after 6 years of Bisphosphonate use.

The American Society for Bone and Mineral Research (ASBMR) published number of criteria in order to identify cases of Bisphosphonate related atypical femoral fractures [8]. Our case fulfilled all of the major criteria. Regarding the minor features, there was periosteal reaction along the lateral cortex, increased cortical thickening and pain starting before evidence of fracture on X-rays.

\section{Conclusion}

Diagnosing bisphosphonates induced AFFs in patient with bony metastasis can be challenging and 
overlooked. Clinical oncologists should consider the possibility of AFFs in patients on long-term Bisphosphonates treatment for bone metastases. Inability to identify those lesions as different from original malignant condition can lead to delayed diagnosis, sub-optimal treatment and missing opportunity to prevent progression of incomplete to complete fractures.

Contributors: IM: manuscript writing, literature search; ME, EH: manuscript editing, literature search. IM will act as guarantor. All authors approved the final version of the manuscript.

Funding: None; Competing interests: None stated.

\section{References}

1. Coleman RE, McCloskey EV. Bisphosphonates in oncology. Bone. 2011;49:71-76.

2. Hillner BE, Ingle JN, Chlebowski RT, Gralow J, Yee GC, Janjan NA, et al. American Society of Clinical Oncology 2003 update on the role of bisphosphonates and bone health issues in women with breast cancer. J Clin Oncol.
2003;21:4042-4057.

3. Diel IJ, Bergner R, Grotz KA. Adverse effects of bisphosphonates: current issues. J Support Oncol. 2007;5:475-482.

4. Drake MT, Clarke BL, Khosla S. Bisphosphonates: mechanism of action and role in clinical practice. Mayo Clin Proc. 2008;83:1032-1045.

5. Aspenberg P. Denosumab and atypical femoral fractures. Acta Orthop. 2014;85(1):1.

6. Odvina CV, Zerwekh JE, Rao DS, Maalouf N, Gottschalk FA, Pak CYC. Severely suppressed bone turnover: a potential complication of alendronate therapy. J Clin Endocrinol Metab. 2005;90:1294-1301.

7. Kendler D, Roux C, Benhamou C, Brown JP, Lillestol $\mathrm{M}$, Siddhanti $\mathrm{S}$, et al. Effects of denosumab on bone mineral density and bone turnover in postmenopausal women transitioning from alendronate therapy. J Bone Miner Res. 2010;25:72-81.

8. Shane E, Burr D, Abrahamsen B, Adler RA, Brown TD, Cheung AM, et al. Atypical subtrochanteric and diaphyseal femoral fractures: second report of a task force of the american society for bone and mineral research: AFF task force report. J Bone Miner Res. 2014;29:1-23. 